\title{
Circ_0000511 accelerates the proliferation, migration and invasion, and restrains the apoptosis of breast cancer cells through the miR-326/TAZ axis
}

\author{
DI WU*, HONGYAO JIA*, ZHIRU ZHANG and SIJIE LI \\ Department of Breast Surgery, The First Hospital of Jilin University, Changchun, Jilin 130021, P.R. China
}

Received August 1,2020; Accepted November 27, 2020

DOI: $10.3892 /$ ijo.2021.5181

\begin{abstract}
Circular RNA 0000511 (circ_0000511) has been observed to be dysregulated in breast cancer (BC). However, the functions of circ_0000511 in breast cancer remain unknown. The expression levels of circ_0000511, ribonuclease P RNA component H1, microRNA-326 (miR-326) and transcriptional co-activator with PDZ-binding motif (TAZ) were examined by reverse transcription-quantitative PCR. Colony formation and MTT assays were conducted to analyze the cellular proliferative ability. The apoptotic rate was assessed by flow cytometry. Western blot analysis was used to evaluate the expression levels of B cell leukemia/lymphoma 2 (Bcl-2), Bcl-2 associated $\mathrm{X}$ apoptosis regulator, cleaved caspase-3 and TAZ. Transwell assays were performed to evaluate the migration and invasion of BC cells. The target interaction between miR-326 and circ_0000511 or TAZ was confirmed by dual-luciferase reporter assay. Xenograft assay was used to identify the function of circ_0000511 in vivo. Circ_0000511 abundance was abnormally elevated in BC tissue samples and cell lines compared with in matched normal cases. Circ_0000511 interference suppressed the proliferation, migration and invasion, and induced apoptosis of BC cells. miR-326 was a direct target of circ_0000511, and circ_0000511 silencing-mediated effects in BC cells were largely reversed by the knockdown of miR-326. miR-326 directly bound to TAZ mRNA, and TAZ accumulation largely attenuated miR-326 overexpression-induced effects in BC cells. Circ_0000511 upregulated the expression levels of TAZ partly via targeting miR-326 in BC cells. Circ_0000511 silencing restrained tumor growth in vivo. Circ_0000511 accelerated the proliferation, migration and invasion, while inhibiting the apoptosis of $\mathrm{BC}$ cells
\end{abstract}

Correspondence to: Dr Sijie Li, Department of Breast Surgery, The First Hospital of Jilin University, 71 Xin min Street, Changchun, Jilin 130021, P.R. China

E-mail: gbgvnc@163.com

*Contributed equally

Key words: breast cancer, circular RNA 0000511, microRNA-326, transcriptional co-activator with PDZ-binding motif through upregulating TAZ expression via sponging miR-326. The circ_0000511/miR-326/TAZ axis may be a novel therapeutic target for BC treatment.

\section{Introduction}

Breast cancer (BC) is a common malignancy that threatens the health of women globally, and it has been predicted that the incidence of $\mathrm{BC}$ will reach $\sim 3.2$ million new cases per year by 2050 globally (1). The heterogeneity of BC in genetic and epigenetic alterations limits its progression of treatment development $(2,3)$. Deep understanding of the molecular mechanisms behind $\mathrm{BC}$ progression is pivotal for identifying novel targets for diagnosis, therapy and prognosis of BC.

Circular RNAs (circRNAs) form covalently continuous circular structures without 3'- or 5'-ends $(4,5)$. CircRNAs regulate cellular physiological $(6,7)$ and pathological $(8,9)$ processes by acting as transcription modulators and microRNA (miRNA/miR) sponges (10). There are increasing studies focusing on the miRNA sponge role of circRNAs in cancer (11-13). Through sponging and sequestering miRNAs, the downstream mRNAs are released from miRNA inhibition, causing the upregulation of mRNAs (13). For instance, Ding et al (14) revealed that circ-ATP8A2 accelerated the progression of cervical cancer via sponging miR-433 to upregulate EGFR expression. Sun et al (15) found that circ-SFMBT2 facilitated gastric cancer progression via acting as a sponge for miR-182-5p to elevate the abundance of cAMP responsive element binding protein 1 . In the present study, the expression pattern and function of circ_0000511 in BC development was investigated.

miRNAs exert pro-tumor or antitumor roles in cancer through modulating the expression levels of mRNAs via degrading them or blocking their translation $(16,17)$. miR-326 has been reported to suppress the progression of $\mathrm{BC}$ via regulating ErbB/PI3K signaling (18). In the present study, the working mechanism of miR-326 in BC progression was investigated.

Transcriptional co-activator with PDZ-binding motif (TAZ) was identified for the first time due to its interaction with 14-3-3 proteins (19). In addition to the interaction with 14-3-3, TAZ was found to interact with SLC9A3 regulator 2, translation elongation factor EF-1a and paired box 3 (19-21). Chan et al (22) have identified the promoting roles of TAZ 
in the migration and invasion of BC cells. However, the molecular mechanism behind TAZ in the progression of $\mathrm{BC}$ remains to be elucidated.

The present study analyzed the influences of circ_0000511 on the malignant phenotypes of $\mathrm{BC}$ cells, and the circ_0000511-miRNA-mRNA axis in BC cells was explored to illustrate its working mechanism.

\section{Materials and methods}

Tissue samples. BC tissue specimens and adjacent non-tumor tissue specimens ( $5 \mathrm{~cm}$ from tumor tissues) were collected from 50 female patients (median age, 53 years; age range, 29-86 years) with BC at The First Hospital of Jilin University (Changchun, China) between March 2016 and November 2017. The exclusion criteria were as follows: i) Patients who received preoperative treatment, including chemotherapy or radiotherapy; and ii) patients who had other types of tumor in other organs. The inclusion criteria were as follows: i) All clinicopathological diagnoses were confirmed by two pathologists; ii) none of the patients received any treatments before surgery; iii) no history of other synchronous malignancies; and iv) no death in the perioperative period. All samples were immediately frozen in liquid nitrogen. All participants provided written informed consent before surgical resection. The present study was approved by the Ethics Committee of The First Hospital of Jilin University.

Cell culture. A panel of human BC cell lines (MCF-7, SK-BR-3, MDA-MB-231 and MDA-MB-468) and a human breast epithelial cell line (MCF-10A) were purchased from BeNa Culture Collection. BC cell lines and MCF-10A cell line were cultured with RPMI-1640 (MCF-10A cell line) or DMEM (BC cell lines) (both from Gibco; Thermo Fisher Scientific, Inc.) supplemented with 10\% FBS (Gibco; Thermo Fisher Scientific, Inc.) and $1 \%$ penicillin (100 units $/ \mathrm{ml}) / \mathrm{strep}$ tomycin $(100 \mu \mathrm{g} / \mathrm{ml})$ mixture (Beijing Solarbio Science \& Technology Co., Ltd.) at $37^{\circ} \mathrm{C}$ with $5 \% \mathrm{CO}_{2}$.

Reverse transcription-quantitative PCR (RT-qPCR). RNA samples were isolated from tissues and cells using TRIzol ${ }^{\circledR}$ (Invitrogen; Thermo Fisher Scientific, Inc.) according to the manufacturer's protocol. RT was conducted using the Bio-Rad iScript kit (Bio-Rad Laboratories, Inc.) and the TaqMan RT kit (Applied Biosystems; Thermo Fisher Scientific, Inc.), according to the manufacturer's protocol, to obtain cDNA, which was later used as the template for qPCR. The amplification of circ_0000511, ribonuclease P RNA component H1 (RPPH1) and TAZ was performed using iQSYBR-Green SuperMix (Bio-Rad Laboratories, Inc.), while the PCR reaction of miR-326 was conducted using the TaqMan MicroRNA assay kit (Applied Biosystems; Thermo Fisher Scientific, Inc.). RT-qPCR started at $95^{\circ} \mathrm{C}$ for $5 \mathrm{~min}$, followed by 40 cycles at $95^{\circ} \mathrm{C}$ for $30 \mathrm{sec}$, $60^{\circ} \mathrm{C}$ for $40 \mathrm{sec}$ and $72^{\circ} \mathrm{C}$ for $30 \mathrm{sec}$. GAPDH (for circ_0000511, RPPH1 and TAZ) and U6 (for miR-326) served as the internal references. The quantification was performed with the $2^{-\Delta \Delta C q}$ method (23). The specific primers are displayed in Table I.

RNase $R$ treatment. RNase $\mathrm{R}$ digestion was used to test the stability and circular structure of circ_0000511 and RPPH1
mRNA. RNA was isolated from MCF-7 and MDA-MB-468 cells using TRIzol ${ }^{\circledR}$ (Invitrogen; Thermo Fisher Scientific, Inc.), and $10 \mu \mathrm{g}$ RNA was digested with $40 \mathrm{U}$ RNase R (Epicentre; Illumina, Inc.). The enrichment of circ_0000511 and RPPH1 mRNA was detected by RTq-PCR, as aforementioned.

Cell transfection. Small interfering (si)RNA of circ_0000511 (si-circ_0000511), scrambled siRNA negative control (si-NC), short hairpin (sh)RNA of circ_0000511 using pGIPZ vector (sh-circ_0000511), pGIPZ empty vector (sh-NC), miR-326 mimics (miR-326), miR-NC, miR-326 inhibitor (anti-miR-326), anti-miR-NC, TAZ overexpression plasmid (TAZ) and pcDNA empty vector (pcDNA) were purchased from Shanghai GenePharma Co., Ltd. The sequences were as follows: si-circ_0000511 sense, 5'-AAUGGCUGAGGU GAGUUCCCA-3' and antisense, 5'-GGAACUCACCUCAGC CAUUGG-3'; si-NC sense, 5'-AACAGGCACACGUCCCAG CGU-3' and antisense, 5'-ACGCUGGGACGUGUGCCU GUU-3'; miR-326, 5'-AGGUCUGGACCGUAAGUCAG-3'; miR-NC, 5'-UGGACGGACCACUGACAUG-3'; anti-miR-326, 5'-CGUGGAUCGAACGUUGGAC-3'; and anti-miR-NC, 5'-ACGGACGUUGAGACACGGG-3'. A total of $1 \mu \mathrm{g}$ plasmid or $0.5 \mu \mathrm{M}$ oligonucleotides was transfected into $\mathrm{MCF}-7$ or MDA-MB-468 cells using Lipofectamine ${ }^{\circledR} 3000$ (Invitrogen; Thermo Fisher Scientific, Inc.). After transfection for $7 \mathrm{~h}$ at $37^{\circ} \mathrm{C}$, the culture medium was replaced by fresh complete culture medium. BC cells were utilized for further analysis after $24 \mathrm{~h}$ of transfection.

Colony formation assay. A total of $150 \mathrm{MCF}-7$ or MDA-MB-468 cells in the control group and experimental groups were seeded into 6-well plates and allowed to grow for 2 weeks at $37^{\circ} \mathrm{C}$. The culture medium was replenished every 6 days. The visible colonies were immobilized using $4 \%$ poly methanol for $15 \mathrm{~min}$ at room temperature, and stained using $0.5 \%$ crystal violet for $10 \mathrm{~min}$ at room temperature. Colonies containing $>50$ cells were counted under a light microscope.

MTT assay. MCF-7 and MDA-MB-468 cells were seeded into 96-well plates to settle down at $37^{\circ} \mathrm{C}$ overnight. The next day, $\mathrm{BC}$ cells were transfected with plasmids or RNAs, as aforementioned. After transfection for specific intervals $(0,1,2$ or 3 days), BC cells were incubated with $10 \mu$ l MTT (Invitrogen; Thermo Fisher Scientific, Inc.) at $37^{\circ} \mathrm{C}$ for $4 \mathrm{~h}$. A total of $100 \mu \mathrm{l}$ DMSO (Sangon Biotech Co., Ltd.) was added to the wells to dissolve the formazan product. The absorbance was detected at $570 \mathrm{~nm}$.

Flow cytometry. The apoptotic rate of transfected MCF-7 and MDA-MB-468 cells was assessed by flow cytometry. BC cells in the early and late stage of apoptosis were differentiated from normal or necrotic BC cells through double staining with Annexin V-FITC and PI at $37^{\circ} \mathrm{C}$ for $15 \mathrm{~min}$ in the dark (both Beijing Solarbio Science \& Technology Co.,Ltd.). The apoptotic rate was evaluated using FlowJo software (v7.6; FlowJo LLC) on a FC-500 flow cytometer (Beckman Coulter, Inc.).

Western blot assay. After plasmid or RNA transfection, MCF-7 and MDA-MB-468 cells were lysed using cell lysis buffer (Abcam). The BCA method was used to examine 
Table I. Primers used for reverse transcription-quantitative PCR.

\begin{tabular}{lll}
\hline Gene & \multicolumn{1}{c}{ Forward primer $\left(5^{\prime}-3^{\prime}\right)$} & Reverse primer $\left(5^{\prime}-3^{\prime}\right)$ \\
\hline circ_0000511 & CCTCCTTTGCCGGAGCTT & GGTCCACGGCATCTCCTG \\
RPPH1 & GAGCTGAGTGCGTCCTGTC & TCAGGGAGAGCCCTGTTAGG \\
miR-326 & CCTCTGGGCCCTTCCTCCAG & Universal primer \\
TAZ & AGTACCCTGAGCCAGCAGAA & GATTCTCTGAAGCCGCAGTT \\
U6 & CTCGCTTCGGCAGCACA & AACGCTTCACGAATTTGCGT \\
GAPDH & GTGAAGGTCGGAGTCAACGG & GAGGTCAATGAAGGGGTCATTG
\end{tabular}

circ, circular RNA 0000511; RPPH1, ribonuclease P RNA component H1; miR, microRNA; TAZ, transcriptional co-activator with PDZ-binding motif.

the concentrations of different protein samples. Protein samples $(25 \mu \mathrm{g} /$ lane) were separated via $10 \%$ SDS-PAGE and transferred to PVDF membranes (Bio-Rad Laboratories, Inc.). After incubating with 5\% skimmed milk for $1 \mathrm{~h}$ at room temperature, the PVDF membranes were incubated overnight at $4^{\circ} \mathrm{C}$ with specific primary antibodies, including anti-B cell leukemia/lymphoma 2 (anti-Bcl-2; 1:5,000; cat. no. ab185002; Abcam), anti-Bcl-2 associated $\mathrm{X}$ apoptosis regulator (anti-Bax; 1:8,000; cat. no. ab32503; Abcam), anti-cleaved caspase-3 (1:10,000; cat. no. ab2302; Abcam), anti-TAZ (1:5,000; cat. no. ab242313; Abcam) and anti-GAPDH (1:20,000; cat. no. ab181602; Abcam). The next day, an HRP-conjugated secondary antibody (1:5,000; cat. no. ab205718; Abcam) was incubated with the PVDF membranes for $2 \mathrm{~h}$ at room temperature. The immunoreactive protein bands were measured using an enhanced chemiluminescent visualization (ECL) kit (Pierce; Thermo Fisher Scientific, Inc.). Image Lab analysis software (v4.0; Bio-Rad Laboratories, Inc.) was used to analyze the intensities of protein bands.

Transwell assays. Transwell chambers (Costar; Corning, Inc.) were used in the present study. For Transwell migration assays, $200 \mu \mathrm{l}$ cell suspension $\left(1 \times 10^{4} \mathrm{MCF}-7\right.$ and MDA-MB-468 cells in serum-free DMEM) was added to the upper chambers. The lower chambers contained culture medium with $10 \% \mathrm{FBS}$. After incubation at $37^{\circ} \mathrm{C}$ for $24 \mathrm{~h}, \mathrm{BC}$ cells on the upper surface of the membrane were discarded using a cotton swab, and the migrated BC cells on the lower surface of the membrane were stained using $0.5 \%$ crystal violet for $10 \mathrm{~min}$ at room temperature. Five random fields at a magnification of x100 were selected to count the number of migrating cells under a light microscope.

For Transwell invasion assays, the upper chambers were pre-coated with $40 \mu \mathrm{l}$ Matrigel (BD Biosciences) at the dilution of $1: 8$ at $37^{\circ} \mathrm{C}$ for $30 \mathrm{~min}$. The remaining steps were similar as for the Transwell migration assays.

Bioinformatic prediction. The circ_0000511-miRNAs interactions and miR-326-mRNAs interactions were explored using the StarBase database (http://starbase.sysu.edu.cn).

Dual-luciferase reporter assay. To test the direct interaction between miR-326 and circ_0000511 or TAZ mRNA, the wild-type (wt) partial sequences in circ_0000511 and TAZ mRNA 3'-untranslated region (UTR), containing the putative binding sequence with miR-326, were amplified and constructed in the psiCHECK-2 luciferase vector (Promega Corporation), generating circ_0000511-wt and TAZ-wt. Meanwhile, circ_0000511-mutant (mut) and TAZ-mut luciferase plasmids containing the mutant binding sites with miR-326 were generated using GeneArtä Site-Directed Mutagenesis System (Invitrogen; Thermo Fisher Scientific, Inc.). Subsequently, the constructed luciferase plasmids were co-transfected into BC cells with miR-NC or miR-326 using Lipofectamine 3000, as aforementioned. Luciferase activities were measured after $24 \mathrm{~h}$ of transfection using the Dual Luciferase Reporter Assay kit (Promega Corporation). The firefly luciferase activity was normalized to Renilla luciferase activity.

Xenografts. A total of 10 female 4-week-old BALB/c nude mice (weight, 16-20 g) from Orient Bio, Inc., were randomly divided into two groups with 5 mice in each group. Mice were maintained under pathogen-free conditions at $22 \pm 2^{\circ} \mathrm{C}$ and $60 \%$ humidity with a 12 -h light/dark cycle, and were supplied with food and water ad libitum. The weight of mice was measured twice a week to monitor the health of mice. Mice weight in the two groups exhibited no significant difference during the experiment. The maximum volume of xenograft tumor was $<1,000 \mathrm{~mm}^{3}$. The mice were euthanized by carbon dioxide suffocation. The $\mathrm{CO}_{2}$ displacement rate used for mouse euthanasia was $30 \%$ chamber volume $/ \mathrm{min}$. The MCF-7 cell line stably transfected with sh-NC or sh-circ_0000511 was collected and re-suspended in PBS buffer. A total of $200 \mu \mathrm{l}$ cell suspension containing $2 \times 10^{6} \mathrm{MCF}-7$ cells was subcutaneously injected into the right flank of nude mice. The length and width of tumor masses were detected every week until 4 weeks after injection when the mice were euthanized. The tumor volume was analyzed with the following formula: Volume $=$ width $^{2} \mathrm{x}$ length $\mathrm{x} 0.5$. The tumors were weighed after removing the tumors from the mice. The abundance of circ_0000511, miR-326 and TAZ mRNA and protein was examined by RT-qPCR and western blot assay, respectively. Animal manipulation was approved by the Research Ethics Committee of The First Hospital of Jilin University.

Statistical analysis. The results were analyzed using GraphPad Prism software (v7.0; GraphPad Software, Inc.) and the data 

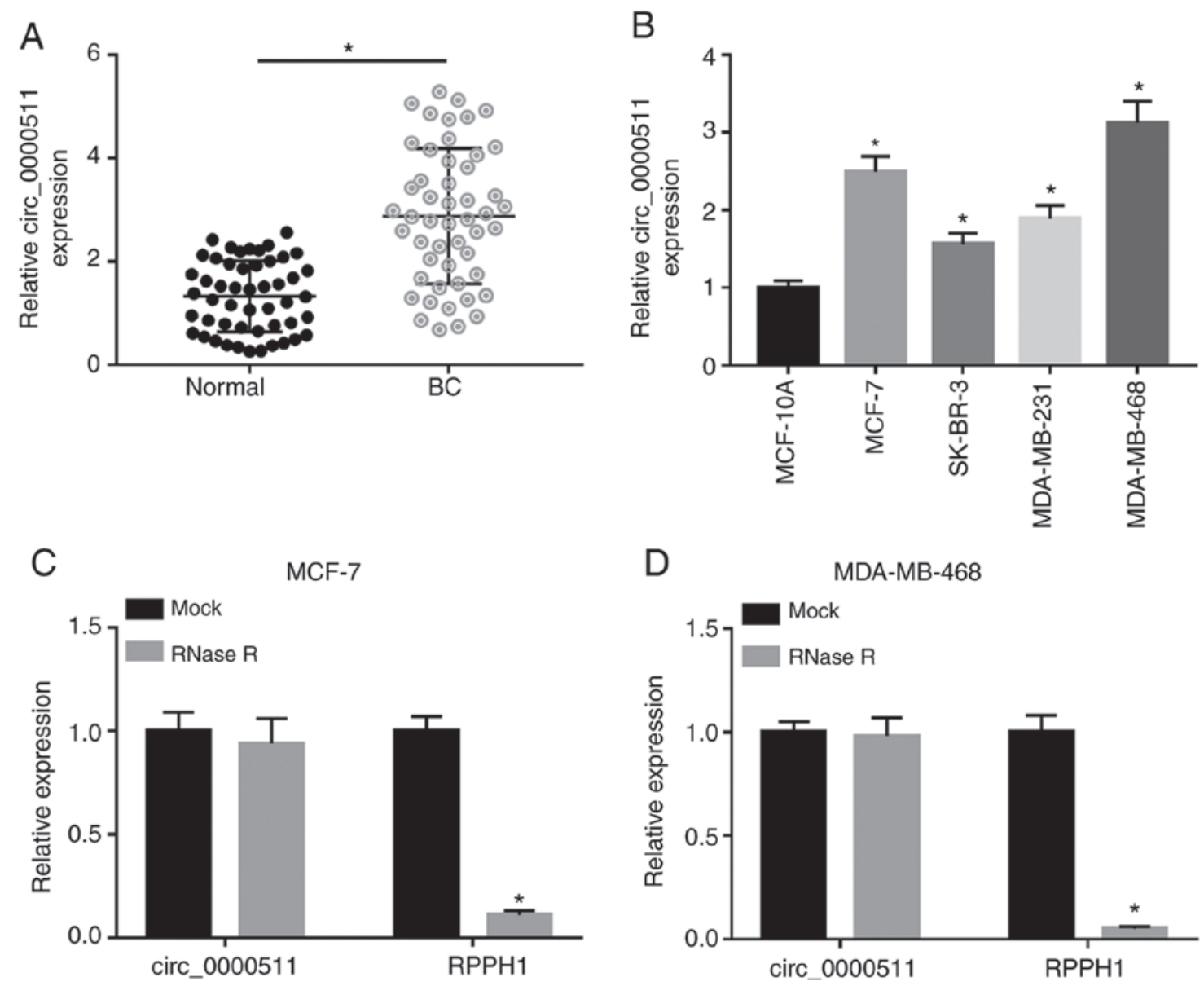

Figure 1. Circ_0000511 expression is upregulated in BC. (A) A total of 50 pairs of BC tissues and adjacent normal tissues were collected to detect the abundance of circ_0000511 by RT-qPCR. (B) Normal breast epithelial MCF-10A cell line and a panel of BC cell lines (MCF-7, SK-BR-3, MDA-MB-231 and MDA-MB-468) were used to measure the abundance of circ_0000511 by RT-qPCR. RNA samples extracted from (C) MCF-7 and (D) MDA-MB-468 cells were digested with RNase R or not, and circ_0000511 and RPPH1 mRNA expression was examined by RT-qPCR. "P $<0.05$ vs. MCF-10A or mock. RT-qPCR, reverse transcription-quantitative PCR; circ_0000511, circular RNA 0000511; BC, breast cancer; RPPH1, ribonuclease P RNA component H1.

are displayed as the mean $\pm \mathrm{SD}$. All in vitro experiments were repeated three times. Differences between BC tissues and adjacent normal tissues were analyzed using paired Student's t-test, while unpaired Student's t-test was utilized to assess the differences between two groups of BC cells. One-way ANOVA followed by Tukey's test was used to analyze the differences in multiple groups. Linear correlation analysis was performed using Spearman's correlation coefficient. $\mathrm{P}<0.05$ was considered to indicate a statistically significant difference.

\section{Results}

Circ_0000511 expression is upregulated in BC.Circ_0000511 expression was significantly elevated in $\mathrm{BC}$ tissues compared with in matching adjacent normal tissues (Fig. 1A). The expression levels of circ_0000511 in the normal breast epithelial MCF-10A cell line and four BC cell lines (MCF-7, SK-BR-3, MDA-MB-231 and MDA-MB-468) were detected by RT-qPCR. As shown in Fig. 1B, circ_0000511 expression was significantly enhanced in $\mathrm{BC}$ cell lines compared with in the MCF-10A cell line. Moreover, to test the stability and loop structure of circ_0000511, exonuclease RNase R was used in subsequent experiments to digest linear RNA but not circular RNA, and the linear form of circ_0000511 (RPPH1 mRNA) was used as the control. RNA samples isolated from BC cells were equally divided into two parts, and these two parts were exposed to RNase R or not. As shown in Fig. 1C and D, RNase R addition significantly downregulated the expression levels of RPPH1, while the abundance of circ_0000511 remained almost unchanged in the RNase R treatment and no treatment groups, suggesting the loop structure of circ_0000511. Overall, circ_0000511 was identified as a circular RNA, and its expression was upregulated in $\mathrm{BC}$ tissues and cell lines.

Circ_0000511 accelerates the proliferation, migration and invasion, and impedes the apoptosis of BC cells. si-circ_0000511 was designed to silence circ_0000511 in BC cell lines to test the biological effects of circ_0000511. Circ_0000511 abundance was significantly downregulated in MCF-7 and MDA-MB-468 cells transfected with si-circ_0000511 compared with the si-NC-transfected group (Fig. 2A). MCF-7 and MDA-MB-468 cell lines were chosen for loss-of-function experiments due to their higher expression of circ_0000511 compared with the other two BC cell lines (SK-BR-3 and MDA-MB-231). The proliferation of $\mathrm{BC}$ cells was analyzed using colony formation and MTT assays. As shown in Fig. 2B, circ_0000511 silencing significantly decreased the number of colonies, suggesting that 

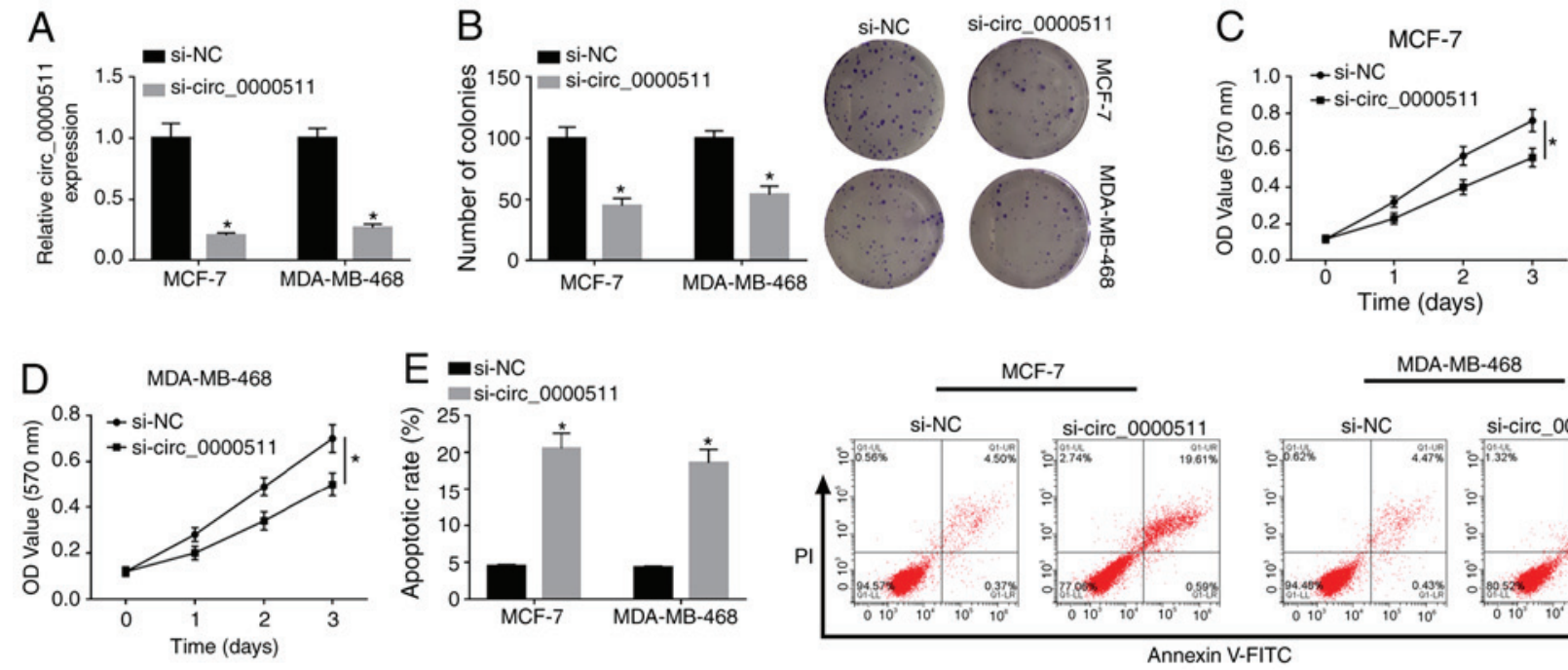

MCF-7

MDA-MB-468
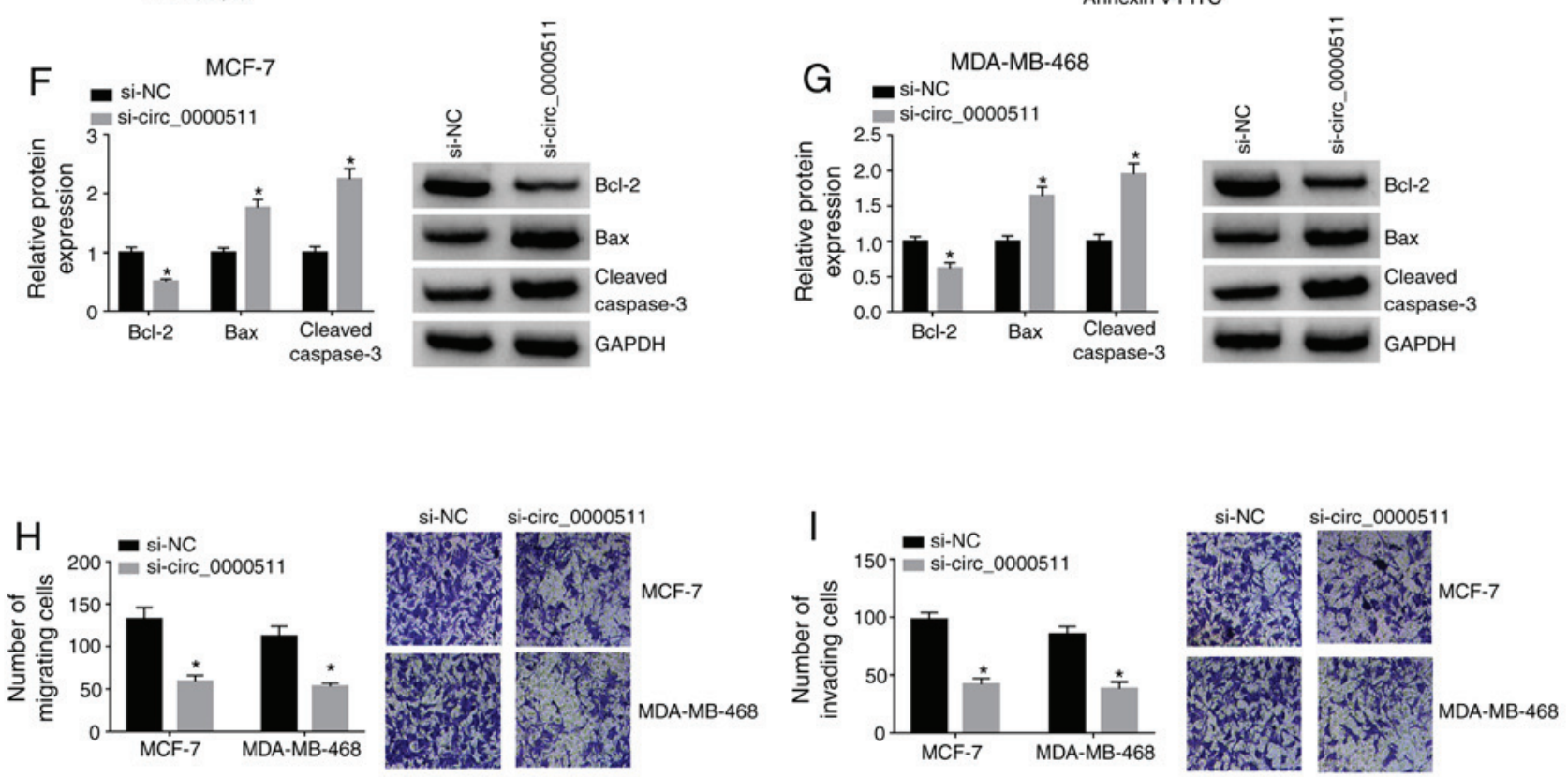

Figure 2. Circ_0000511 accelerates the proliferation, migration and invasion, and impedes the apoptosis of BC cells. MCF-7 and MDA-MB-468 cells were transfected with si-NC or si-circ_0000511. (A) Circ_0000511 expression was detected in BC cells by reverse transcription-quantitative PCR. (B) Colony formation assay was used to test the proliferative ability of BC cells. MTT assay was used to assess the proliferation of (C) MCF-7 and (D) MDA-MB-468 cells. (E) Apoptosis of BC cells was evaluated by flow cytometry. Western blot assay was used to detect the protein expression levels of Bcl-2, Bax and Cleaved caspase-3 in (F) MCF-7 and (G) MDA-MB-468 cells. Transwell assays were conducted to assess the (H) migratory and (I) invasive abilities of BC cells ${ }^{*} \mathrm{P}<0.05$ vs. si-NC. Bcl-2, B cell leukemia/lymphoma 2; Bax, Bcl-2 associated X apoptosis regulator; BC, breast cancer; circ_0000511, circular RNA 0000511; si, small interfering RNA; NC, negative control; OD, optical density.

circ_0000511 interference suppressed the proliferative ability of BC cells. Additionally, the results of MTT assay revealed that circ_0000511-knockdown impaired the proliferation of BC cells (Fig. 2C and D). Furthermore, circ_0000511 interference triggered the apoptosis of BC cells (Fig. 2E). To further clarify the influence of circ_0000511 interference on the apoptosis of $\mathrm{BC}$ cells, western blot assay was conducted to detect the protein expression levels of Bcl-2, Bax and Cleaved caspase-3 in $\mathrm{BC}$ cells. As shown in Fig. 2F and G, the expression levels of the pro-apoptotic proteins (Bax and Cleaved caspase-3) were significantly upregulated in $\mathrm{BC}$ cells with the silencing of circ_0000511, while circ_0000511 interference significantly downregulated the expression levels of the anti-apoptotic protein Bcl-2, demonstrating that circ_0000511-knockdown promoted the apoptosis of $\mathrm{BC}$ cells. The migratory and invasive abilities were both significantly suppressed with the silencing of circ_0000511 compared with in the si-NC group (Fig. 2H and I), suggesting that circ_0000511-knockdown restrained the migration and invasion of $\mathrm{BC}$ cells. Overall, high circ_0000511 expression increased the malignant behaviors of $\mathrm{BC}$ cells.

miR-326 directly interacts with circ_0000511 in BC cells. Considering the miRNA sponge role of circRNAs (13), the present study investigated whether circ_0000511 accelerated the progression of $\mathrm{BC}$ through targeting miRNAs. The complementary binding sites between circ_0000511 and miR-326 predicted using the StarBase database are displayed in Fig. 3A. To test if circ_0000511 interacted with miR-326 through the predicted binding sites, these sites in circ_0000511 were 

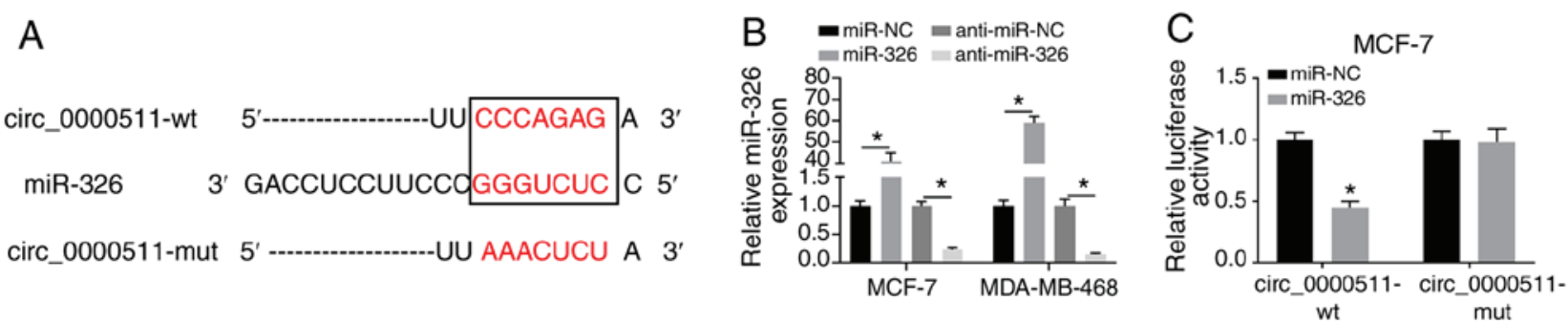
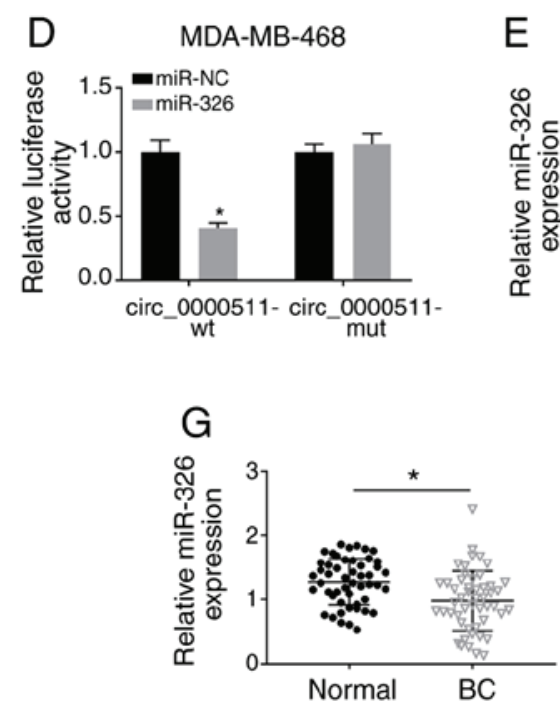
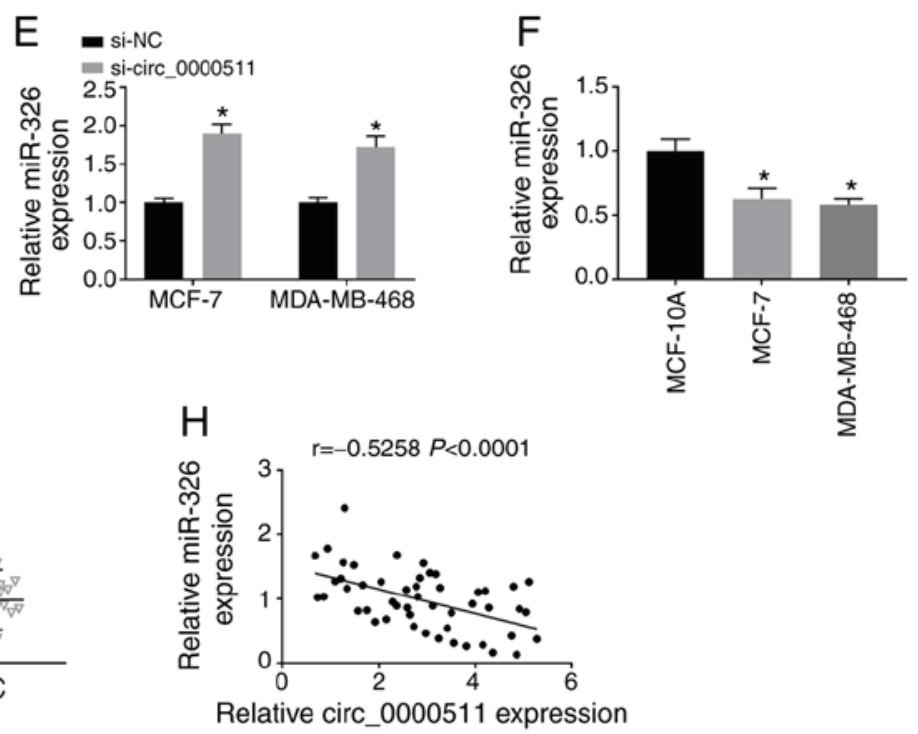

Figure 3. miR-326 directly interacts with circ_0000511 in BC cells. (A) Circ_0000511-miRNAs interactions were explored using StarBase 3.0 software. The paring sites between circ_0000511 and miR-326 are shown, and the complementary site in circ_0000511 was mutated to 'AAACUCU'. (B) miR-326 expression was determined in BC cells transfected with miR-NC, miR-326, anti-miR-NC or anti-miR-326 by RT-qPCR. Dual-luciferase reporter assay was utilized to verify the target association between miR-326 and circ_0000511 in (C) MCF-7 and (D) MDA-MB-468 cells. (E) miR-326 expression in BC cells transfected with si-NC or si-circ_0000511 was detected by RT-qPCR. miR-326 expression was examined in (F) BC cell lines and MCF-10A cells, and (G) BC tissues and paired adjacent normal tissues by RT-qPCR. (H) Linear correlation between miR-326 and circ_0000511 expression was analyzed by Spearman's correlation coefficient. ${ }^{*} \mathrm{P}<0.05$ vs. miR-NC, si-NC or MCF-10A. RT-qPCR, reverse transcription-quantitative PCR; circ_0000511, circular RNA 0000511; BC, breast cancer; miR, microRNA; si, small interfering RNA; NC, negative control; wt, wild-type; mut, mutant.

mutated to 'AAACUCU' (Fig. 3A). The transfection efficiencies of miR-326 and anti-miR-326 were both high in BC cells (Fig. 3B). The partial sequence in circ_0000511, including the wt or mut binding sites with miR-326, was cloned into luciferase vectors, named as circ_0000511-wt or circ_0000511-mut. The luciferase reporter vector was co-transfected with miR-NC or miR-326 into BC cells. As shown in Fig. 3C and D, miR-326 overexpression significantly decreased the luciferase activity in the circ_0000511-wt group compared with in the miR-NC and circ_0000511-wt co-transfected group. However, miR-326 transfection had no influence in luciferase activity in the circ_0000511-mut group compared with in the miR-NC and circ_0000511-mut group (Fig. 3C and D). The results of the dual-luciferase reporter assay demonstrated that miR-326 was a direct target of circ_0000511 in BC cells. Additionally, circ_0000511 silencing significantly upregulated the expression levels of miR-326 in BC cells (Fig. 3E), suggesting a negative regulatory association between miR-326 and circ_0000511 in BC cells. Among three cell lines, including MCF-10A and two BC cell lines, miR-326 expression was significantly decreased in BC cell lines compared with in MCF-10A cells (Fig. 3F). Furthermore, miR-326 expression was significantly downregulated in BC tissues compared with that in normal tissues (Fig. 3G). The analysis of Spearman's correlation coefficient suggested that miR-326 expression was negatively correlated with circ_0000511 expression in BC tissues (Fig. 3H). Overall, the present results indicated that miR-326 was a direct target of circ_0000511 in BC cells.

Circ_0000511 contributes to BC progression via targeting miR-326. Circ_0000511-knockdown significantly upregulated miR-326 expression, which was then significantly decreased by the addition of anti-miR-326 in BC cells (Fig. 4A). Therefore, further rescue experiments were conducted via transfecting si-circ_0000511 alone or together with anti-miR-326 into BC cells. The introduction of anti-miR-326 partly recovered the number of colonies (Figs. 4B and S1A) and the number of viable $\mathrm{BC}$ cells after 3 days of transfection (Fig. 4C and D), demonstrating that anti-miR-326 addition partly recovered the proliferative ability of $\mathrm{BC}$ cells. Meanwhile, the apoptosis of these transfected BC cells was assessed by flow cytometry. Circ_0000511 silencing-induced apoptosis in BC cells was largely counteracted by the introduction of anti-miR-326 (Figs. 4E and S1B). The protein expression levels of Bax and Cleaved caspase-3 were significantly upregulated by the silencing of circ_0000511, while the addition of anti-miR-326 significantly downregulated the expression levels of these two proteins (Fig. 4F and G). The abundance of the anti-apoptotic protein Bcl-2 was significantly downregulated in the si-circ_0000511 group, and the addition of anti-miR-326 

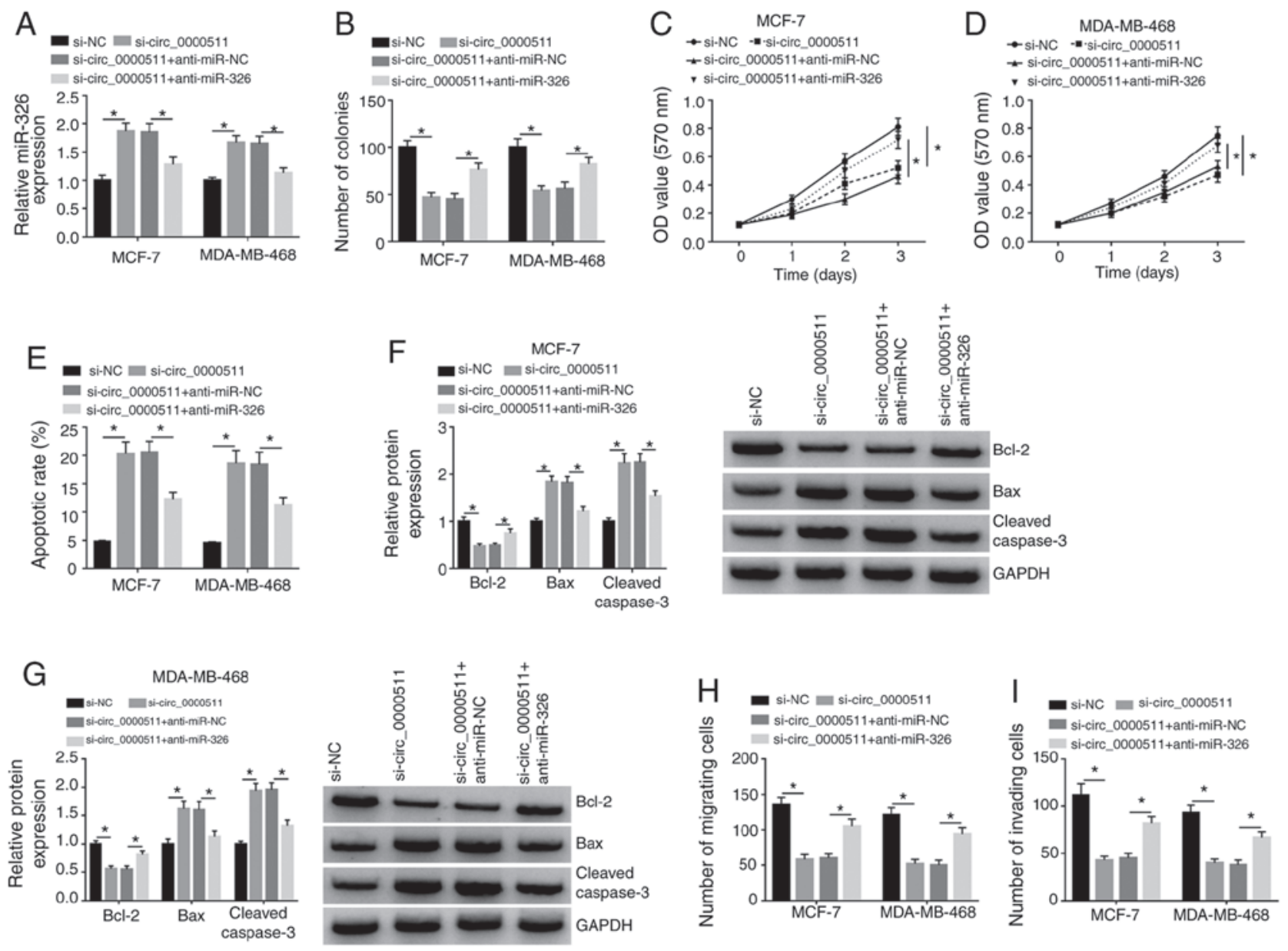

Figure 4. Circ_0000511 contributes to BC progression via targeting miR-326. MCF-7 and MDA-MB-468 cells were transfected with the following four groups: si-NC, si-circ_0000511, si-circ_0000511 + anti-miR-NC or si-circ_0000511 + anti-miR-326. (A) miR-326 expression in the two transfected BC cell lines was examined by reverse transcription-quantitative PCR. (B) Proliferation of BC cells was analyzed using colony formation assay. MTT assay was utilized to assess the proliferation ability of (C) MCF-7 and (D) MDA-MB-468 cells. (E) Apoptotic rate in these four groups was analyzed by flow cytometry. Western blot assay was utilized to detect the protein expression of Bcl-2, Bax and Cleaved caspase-3 in (F) MCF-7 and (G) MDA-MB-468 cells. (H) Migration and (I) invasion of $\mathrm{BC}$ cells were analyzed using Transwell assays (magnification, $\mathrm{x} 100$ ). " $\mathrm{P}<0.05$. Bcl-2, B cell leukemia/lymphoma 2; Bax, Bcl-2 associated X apoptosis regulator; circ_0000511, circular RNA 0000511; BC, breast cancer; miR, microRNA; si, small interfering RNA; NC, negative control; OD, optical density.

largely recovered Bcl-2 expression (Fig. $4 \mathrm{~F}$ and $\mathrm{G}$ ). The present findings further demonstrated that circ_0000511 restrained the apoptosis of $\mathrm{BC}$ cells via targeting miR-326. Furthermore, migration and invasion were suppressed with the silencing of circ_0000511, and the introduction of anti-miR-326 largely rescued the migratory and invasive abilities of $\mathrm{BC}$ cells (Figs. 4H and I, and S1C and D). Overall, circ_0000511 accelerated the proliferation, migration and invasion, and inhibited the apoptosis of BC cells via sponging miR-326.

miR-326 directly binds to TAZ mRNA in BC cells. miRNAs are implicated in the pathogenesis of cancer partly through downregulating the expression levels of target mRNAs associated with cellular proliferation, apoptosis, migration and invasion $(16,17)$. miR-326-mRNA interactions were searched using the StarBase 3.0 database. The complementary sequence between TAZ mRNA and miR-326 is shown in Fig. 5A. The site-directed mutation in TAZ is also shown in Fig. 5A. MCF-7 and MDA-MB-468 cells were co-transfected with miR-NC or miR-326 and TAZ-wt or TAZ-mut, and the luciferase activities in these four transfected groups were detected using the dual-luciferase reporter assay. Compared with the miR-NC and
TAZ-wt co-transfected group, miR-326 transfection significantly decreased the relative luciferase activity in the TAZ-wt group (Fig. 5B and C). However, miR-NC or miR-326 transfection did not affect the luciferase activity in the TAZ-mut groups (Fig. 5B and C), suggesting a direct association between miR-326 and TAZ mRNA in BC cells. The present study further explored the regulatory association between miR-326 and TAZ by detecting the mRNA and protein expression levels of TAZ in BC cells transfected with miR-NC, miR-326, anti-miR-NC or anti-miR-326. miR-326 overexpression significantly downregulated the mRNA and protein expression levels of TAZ, while the inhibition of miR-326 significantly upregulated TAZ mRNA and protein expression (Fig. 5D and E). The current findings suggested that TAZ was negatively regulated by miR-326 in BC cells. The expression feature of TAZ and the expression correlation between TAZ and miR-326 were tested in BC. As shown in Fig. 5F and G, TAZ mRNA and protein expression was significantly upregulated in $\mathrm{BC}$ tissues compared with in adjacent normal tissues. The results of Spearman's correlation coefficient analysis revealed that there was a negative correlation between miR-326 and TAZ mRNA expression in BC tissues (Fig. 5H). Additionally, TAZ mRNA 
A

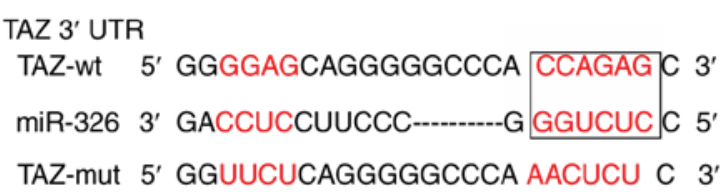

$\mathrm{B}$

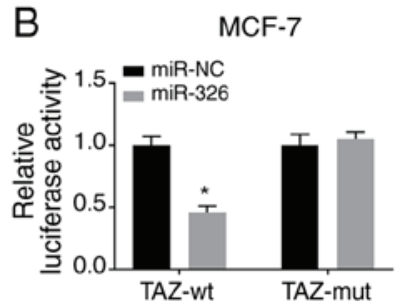

C MDA-MB-468
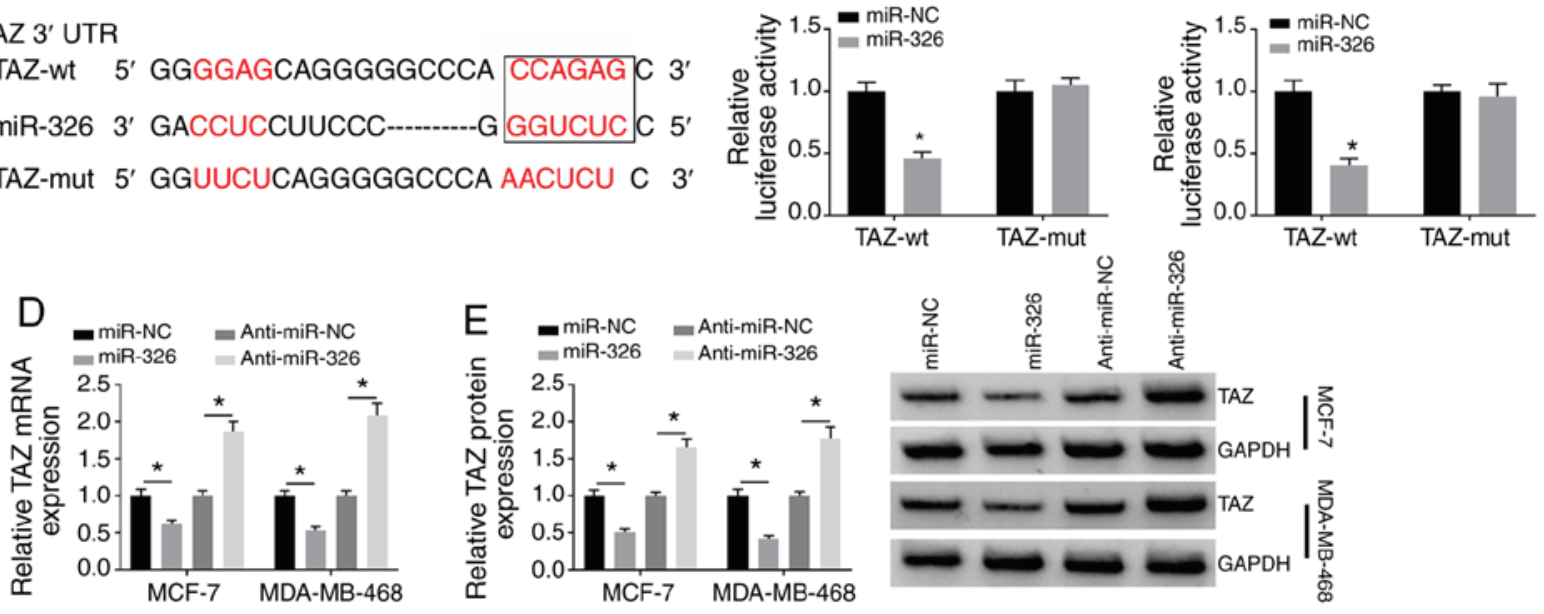

$\mathrm{F}$

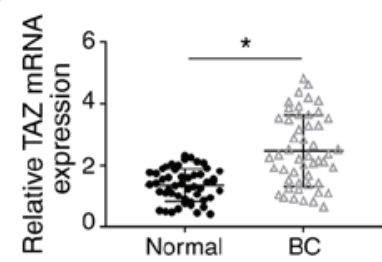

$\mathrm{G}$
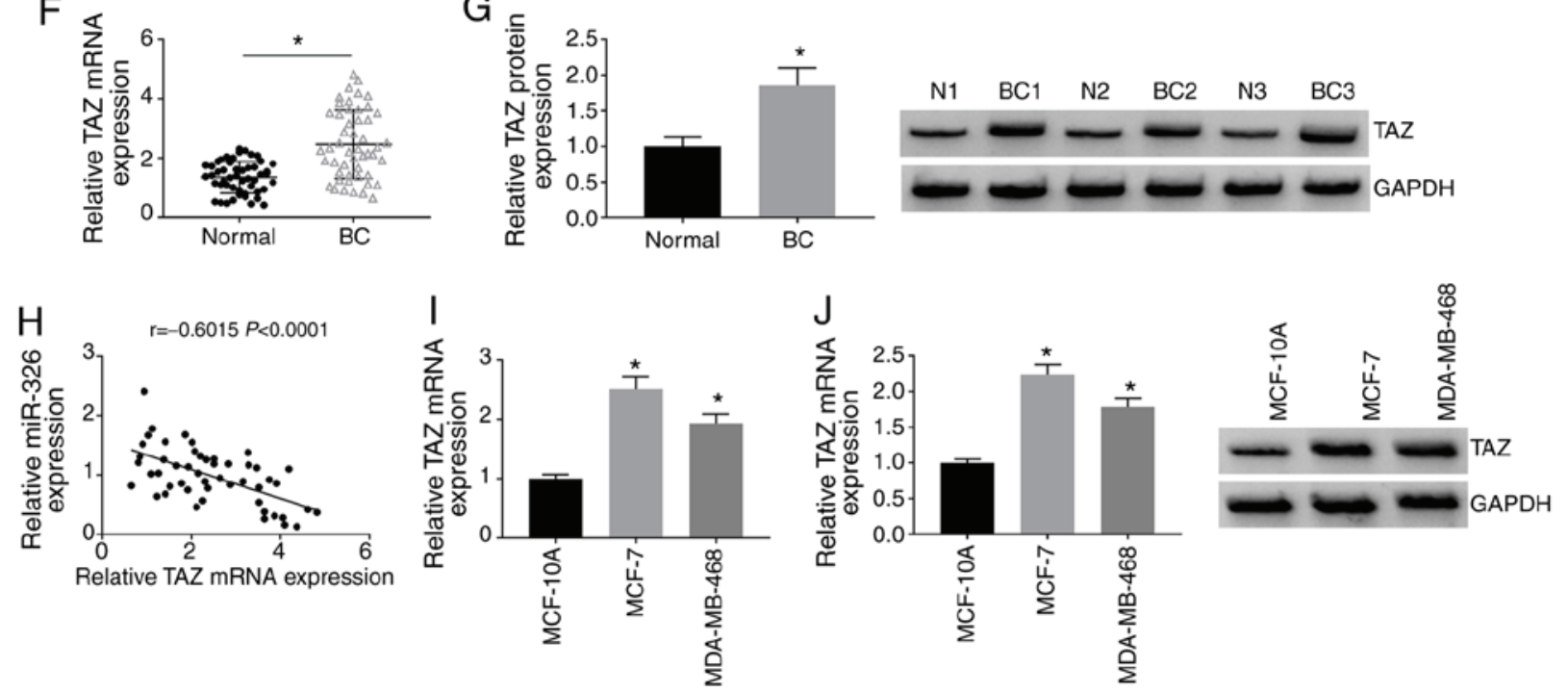

Figure 5. miR-326 directly binds to TAZ mRNA in BC cells. (A) StarBase software was utilized to search miR-326-interacted mRNAs, and TAZ was found as a possible target of miR-326. Luciferase activities in (B) MCF-7 and (C) MDA-MB-468 cells co-transfected with miR-NC or miR-326 and TAZ-wt or TAZ-mut were detected using dual-luciferase reporter assay. MCF-7 and MDA-MB-468 cells were transfected with miR-NC, miR-326, anti-miR-NC or anti-miR-326. TAZ (D) mRNA and (E) protein expression in transfected BC cells was analyzed by RT-qPCR and western blot assay, respectively. TAZ (F) mRNA and (G) protein expression in BC tissues and adjacent normal tissues was detected by RT-qPCR and western blot assay, respectively. (H) Correlation between miR-326 and TAZ mRNA expression was analyzed using Spearman's correlation coefficient. (I) RT-qPCR and (J) western blot assay were implemented to examine the mRNA and protein expression levels of TAZ, respectively, in BC cell lines and MCF-10A cells. * $\mathrm{P}<0.05$ vs. miR-NC, normal or MCF-10A. RT-qPCR, reverse transcription-quantitative PCR; BC, breast cancer; miR, microRNA; si, small interfering RNA; NC, negative control; wt, wild-type; mut, mutant; UTR, untranslated region; TAZ, transcriptional co-activator with PDZ-binding motif; N, normal.

and protein expression was significantly upregulated in BC cell lines compared with in the MCF-10A cell line (Fig. 5I and J). Overall, miR-326 negatively regulated the abundance of TAZ by directly targeting it in BC cells.

miR-326-mediated influences are partly reversed by the overexpression of TAZ in BC cells. The overexpression of TAZ was tested by RT-qPCR assay and western blot assay. As shown in Fig. S2A and B, the mRNA and protein expression levels of TAZ were both significantly upregulated following the transfection of TAZ plasmid in BC cells, suggesting the high transfection efficiency of the TAZ plasmid. miR-326 overexpression significantly downregulated the mRNA and protein expression levels of TAZ in BC cells, and the addition of TAZ overexpression plasmid rescued the $\mathrm{mRNA}$ and protein abundance of TAZ in BC cells (Fig. 6A and B). Therefore, further rescue experiments were conducted by transfecting miR-NC, miR-326, miR-326 + pcDNA or miR-326 + TAZ into BC cells to test if miR-326 functioned via decreasing TAZ expression. miR-326 overexpression suppressed cellular proliferation, migration and invasion, while apoptosis was increased with the overexpression of miR-326 (Fig. 6C-J), which further demonstrated that miR-326 exerted an antitumor role in $\mathrm{BC}$ cells. The addition of TAZ overexpression plasmid partly recovered the proliferative ability of $\mathrm{BC}$ cells according to the results of colony formation assay (Figs. 6C and S3A) and MTT assay (Fig. 6D and E). The results of flow cytometry (Figs. 6F and S3B) and western blot assay (Fig. 6G and H) both suggested that miR-326-induced apoptosis in BC cells was partly counteracted by the overexpression of TAZ. Additionally, the migration and invasion of $\mathrm{BC}$ cells were largely regained in the miR-326 and TAZ co-transfected 

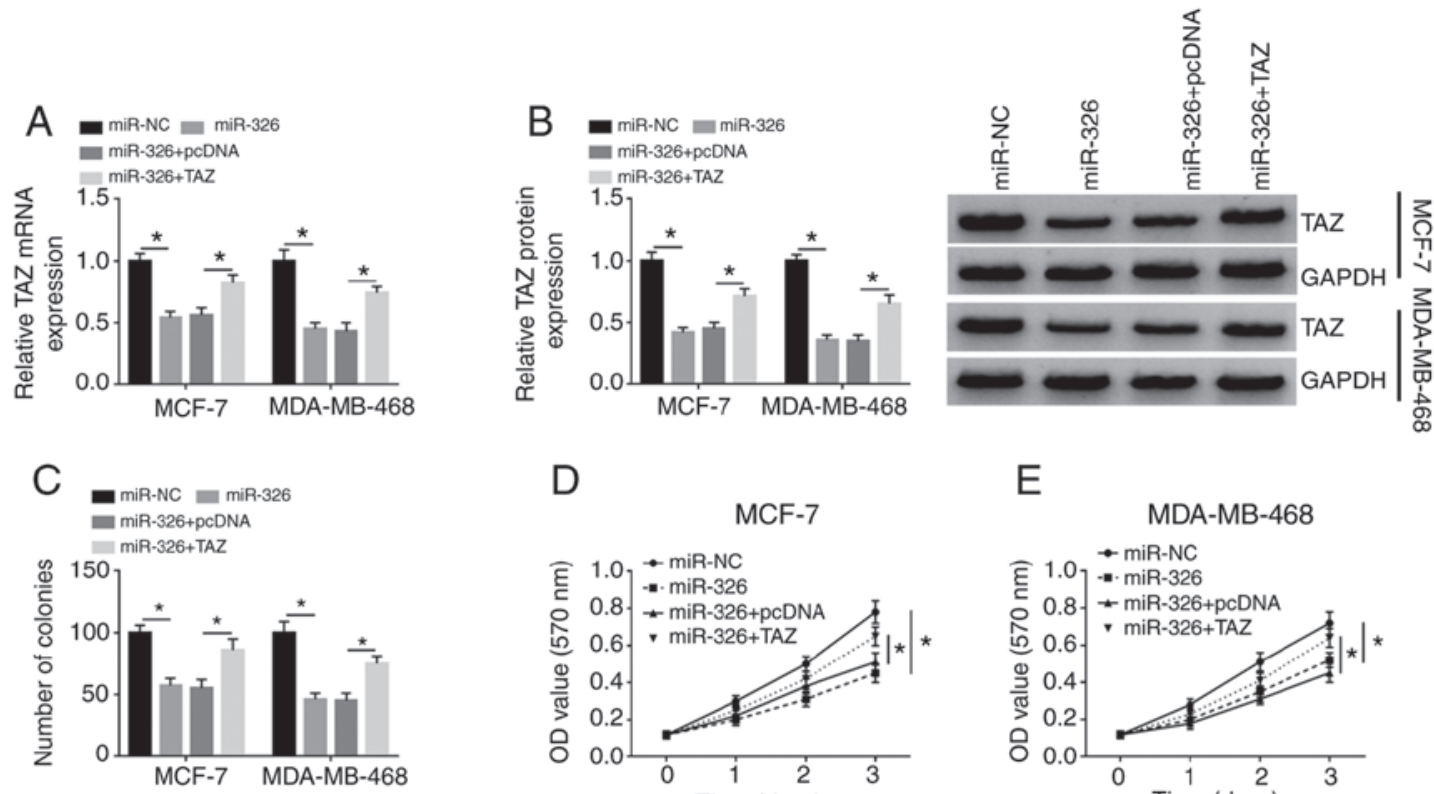

$\mathrm{D}$
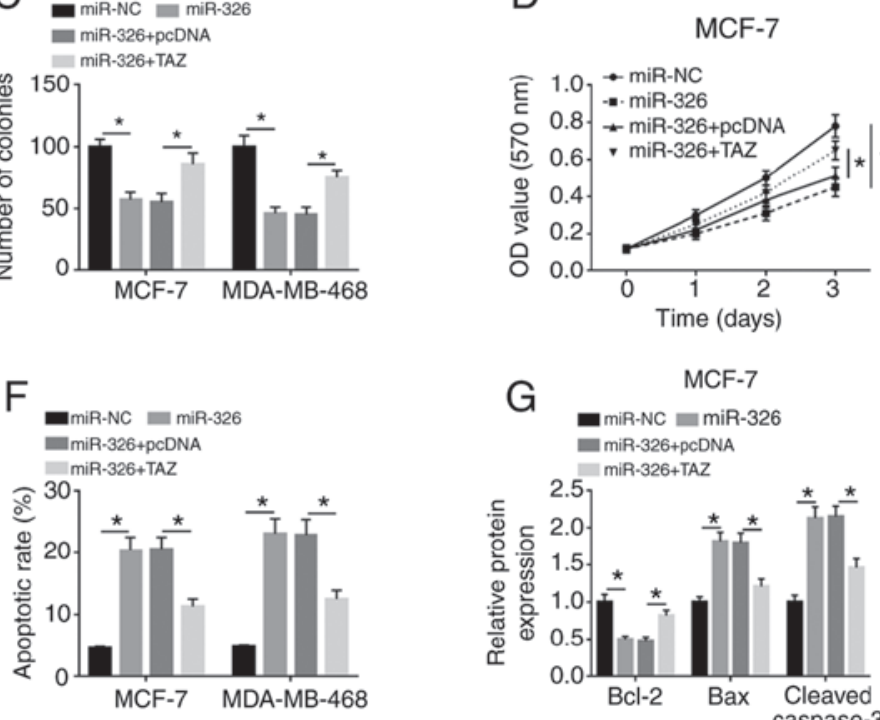

$\mathrm{E}$
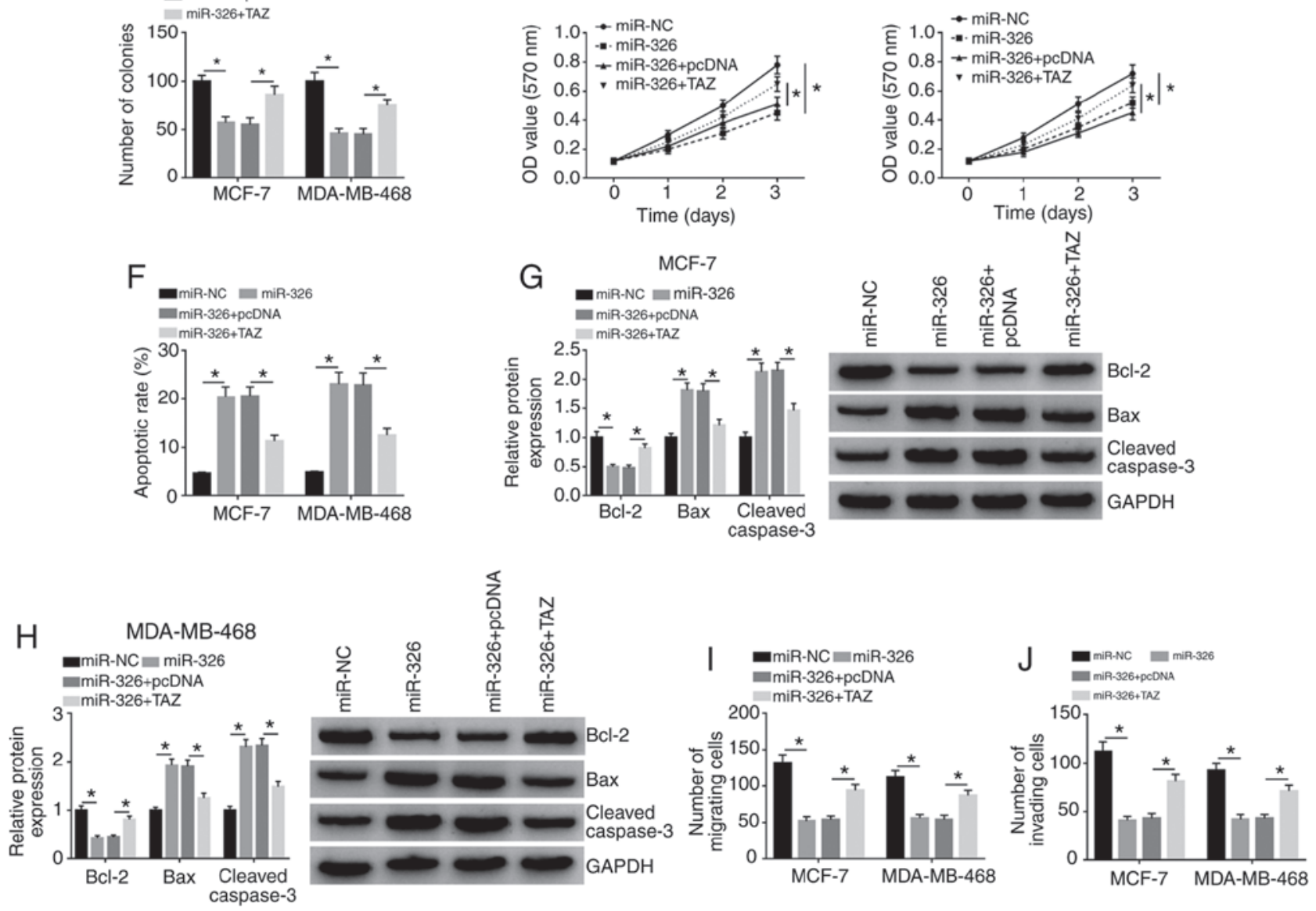

Figure 6. miR-326-mediated influences are partly reversed by the overexpression of TAZ in BC cells. MCF-7 and MDA-MB-468 cells were transfected with miR-NC, miR-326, miR-326 + pcDNA or miR-326 + TAZ. (A) Reverse transcription-quantitative PCR and (B) western blot assay were used to examine the mRNA and protein expression levels of TAZ, respectively, in BC cells. (C) Colony formation assay was performed to measure the proliferative capacity of BC cells, and the number of colonies in each group was detected. MTT assay was used to analyze the proliferative ability of (D) MCF-7 and (E) MDA-MB-468 cells. (F) Flow cytometry was conducted to assess the apoptosis of BC cells. Protein expression levels of Bcl-2, Bax and Cleaved caspase-3 were detected in (G) MCF-7 and (H) MDA-MB-468 cells by western blot assay. Transwell assays were conducted to assess the (I) migration and (J) invasion of BC cells. ${ }^{*} \mathrm{P}<0.05$. Bcl-2, B cell leukemia/lymphoma 2; Bax, Bcl-2 associated X apoptosis regulator; BC, breast cancer; miR, microRNA; NC, negative control; TAZ, transcriptional co-activator with PDZ-binding motif; OD, optical density.

group (Figs. 6I and J, and S3C and D). The current findings demonstrated that miR-326 suppressed the progression of $\mathrm{BC}$ via downregulating TAZ expression.

Circ_0000511 elevates TAZ mRNA and protein expression via sponging miR-326 in BC cells. After identifying the negative regulatory association between miR-326 and circ_0000511 or TAZ, the present study subsequently explored the association among circ_0000511, miR-326 and TAZ in BC cells. MCF-7 and MDA-MB-468 cells were transfected with
si-NC, si-circ_0000511, si-circ_0000511 + anti-miR-NC or si-circ_0000511 + anti-miR-326. The mRNA and protein expression levels of TAZ were analyzed by RT-qPCR and western blot assay, respectively. As shown in Fig. 7A and B, circ_0000511 silencing significantly downregulated the mRNA and protein expression levels of TAZ, and the addition of anti-miR-326 partly recovered the mRNA and protein abundance of TAZ in BC cells, suggesting that circ_0000511 enhanced the mRNA and protein expression levels of TAZ via acting as a sponge of miR-326. 

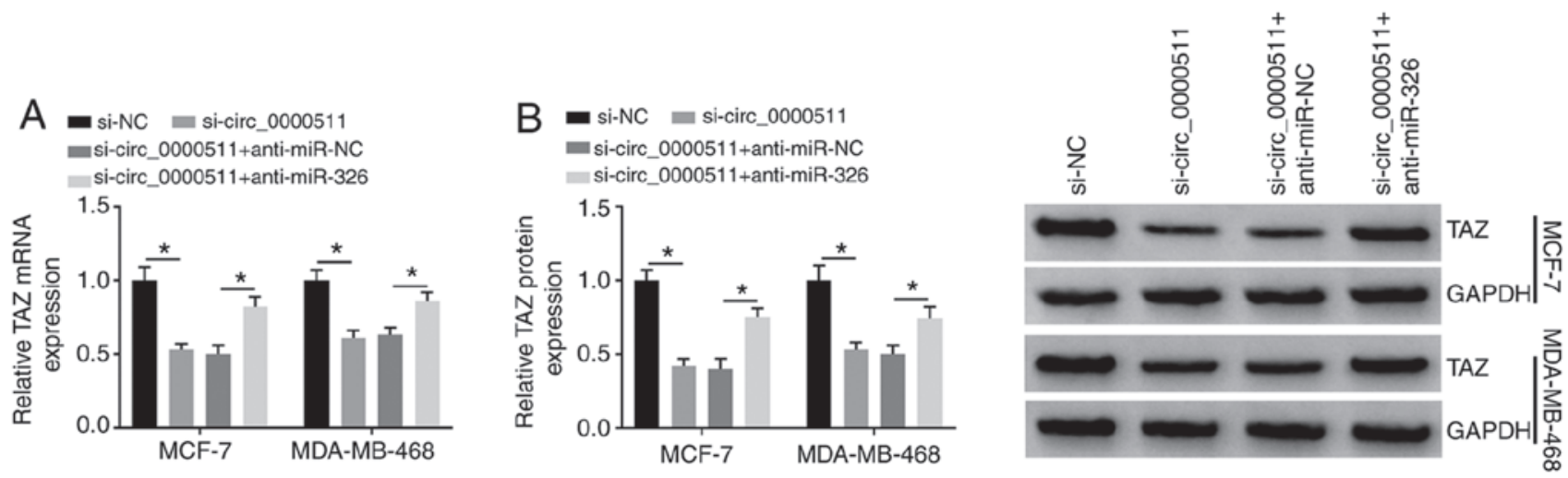

Figure 7. Circ_0000511 elevates TAZ mRNA and protein expression via sponging miR-326 in BC cells. MCF-7 and MDA-MB-468 cells were transfected with si-circ_0000511 alone or together with anti-miR-326. (A) Reverse transcription-quantitative PCR and (B) western blot assay were used to assess the mRNA and protein abundance of TAZ in BC cells. "P<0.05. circ_0000511, circular RNA 0000511; BC, breast cancer; miR, microRNA; si, small interfering RNA; NC, negative control; TAZ, transcriptional co-activator with PDZ-binding motif.
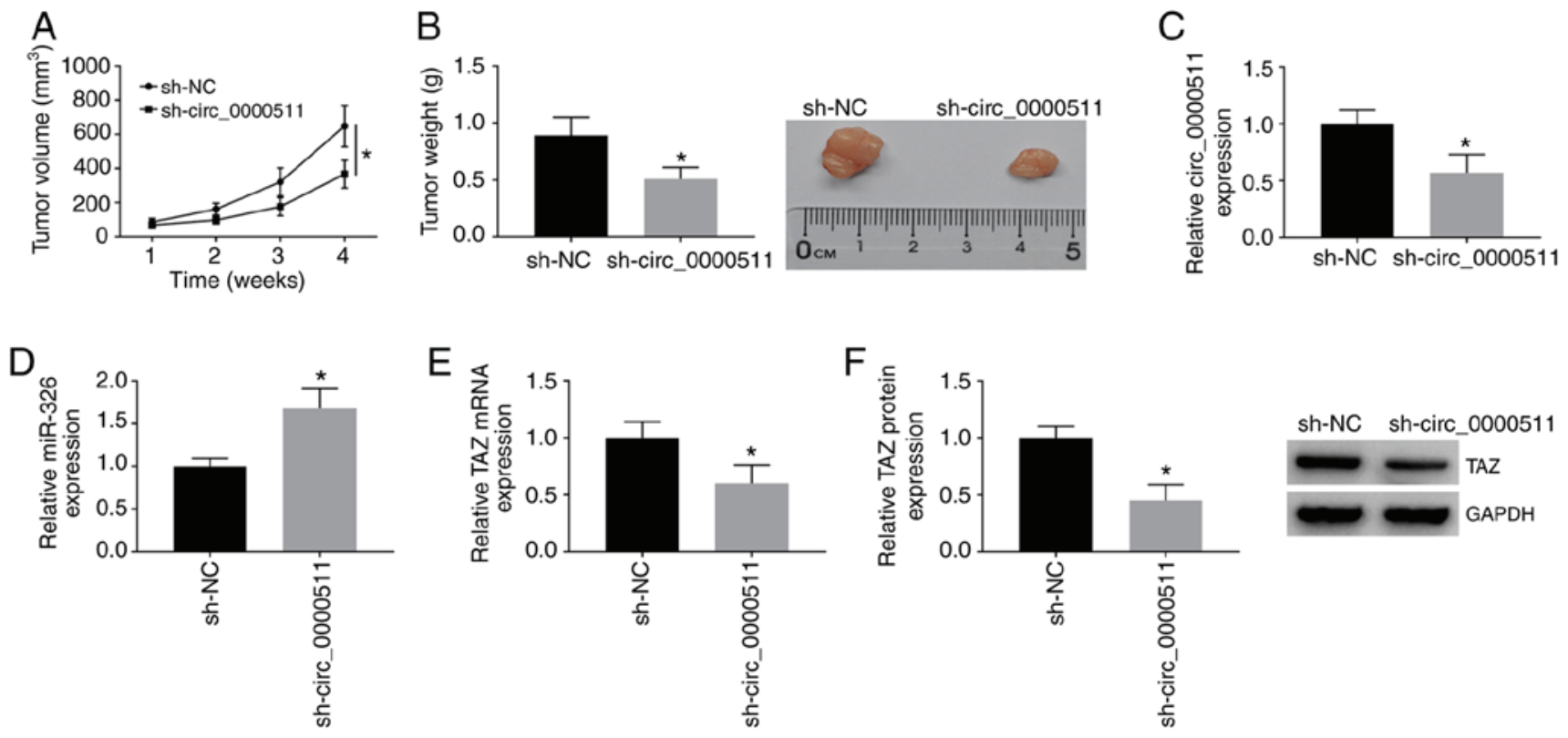

Figure 8. Circ_0000511 promotes BC tumor progression in vivo. MCF-7 cell line was stably transfected with sh-NC or sh-circ_0000511. Nude mice were subcutaneously injected with MCF-7 (2x106 cells/200 $\mu$ l PBS) stably expressing sh-NC or sh-circ_0000511. (A) Tumor volume was measured every week. (B) Mice were euthanized after 4 weeks of inoculation, and the tumors were resected and weighed. (C) Circ_0000511 and (D) miR-326 expression was measured in the sh-NC and sh-circ_0000511 groups by RT-qPCR. (E) RT-qPCR and (F) western blot assay were used to examine the mRNA and protein expression levels of TAZ, respectively, in tumor tissues. "P<0.05 vs. sh-NC. circ_0000511, circular RNA 0000511; BC, breast cancer; miR, microRNA; sh, short hairpin RNA; NC, negative control; TAZ, transcriptional co-activator with PDZ-binding motif.

Circ_0000511 promotes BC tumor progression in vivo. On the basis of the oncogenic role of circ_0000511 in vitro, the present study further assessed the influence of circ_0000511 silencing on the growth of BC tumors in vivo. An MCF-7 cell line stably transfected with sh-NC or sh-circ_0000511 was established. Tumor growth curves were generated by recording the tumor volume every week for 4 weeks. As shown in Fig. 8A, tumors in the sh-NC group were bigger than tumors in the sh-circ_0000511 group. Additionally, by measuring the tumor weight after resecting tumors 4 weeks after inoculation, it was revealed that circ_0000511 silencing suppressed tumor growth in vivo (Fig. 8B). The mRNA and protein expression levels of circ_0000511, miR-326 and TAZ were measured by RT-qPCR and western blot assay, respectively. As shown in Fig. 8C, circ_0000511 abundance was significantly downregulated in the sh-circ_0000511 group compared with that in the sh-NC group. Additionally, miR-326 expression was significantly increased in the sh-circ_0000511 group compared with that in the sh-NC group (Fig. 8D), and TAZ mRNA and protein expression was significantly decreased in the sh-circ_0000511 group compared with that in the sh-NC group (Fig. 8E and F). Overall, the present results suggested that circ_0000511 accelerated the tumor growth of BC in vivo.

\section{Discussion}

CircRNAs have been identified as crucial regulators in the occurrence and development of different types of cancer. 
For instance, circ_0014359 accelerates the development of glioma via modulating the miR-153/PI3K signaling pathway (24). Circ_0020123 contributes to the development of non-small cell lung cancer by upregulating ADAM9 via sponging miR-488-3p (25). In the present study, circ_0000511 expression was higher in BC tissues compared with in adjacent normal tissues, which was consistent with the expression tendency of circ_0000511 in the GSE101124 dataset (26). By performing loss-of-function experiments, the current study revealed that circ_0000511 promoted the proliferation, migration and invasion, and impaired the apoptosis of BC cells.

miRNA sponge mechanism is an important way by which circRNAs function. For instance, circ_0008035 accelerates the proliferation and impedes the apoptosis of gastric cancer cells via sponging miR-599 to upregulate eukaryotic translation initiation factor 4A1 (27). Circ_0079593 deteriorates glioma by promoting the proliferation and motility of glioma cells via sponging miR-182 and miR-433 (28). In the present study, the interaction between circ_0000511 and miR-326 was predicted using a bioinformatic software (StarBase) and then verified by dual-luciferase reporter assay. A previous study has demonstrated that miR-326 acts as a tumor suppressor in $\mathrm{BC}$ cells via regulating the ErbB/PI3K signaling pathway (18). Furthermore, miR-326 exerts an antitumor role in a variety of types of cancer by targeting different downstream mRNAs. For example, Ji et al (29) claimed that miR-326 restrains the progression of gastric cancer via decreasing the abundance of NIN1 binding protein 1 homolog. Liang et al (30) demonstrated that miR-326 suppresses the development of prostatic carcinoma via targeting Mucin1. The results of rescue experiments in the present study demonstrated that circ_0000511 exhibited an oncogenic role in BC cells partly via sponging miR-326. The tumor suppressor role of miR-326 was in agreement with a previous study (18).

The interaction between TAZ mRNA and miR-326 was also predicted using the StarBase database and was validated by dual-luciferase reporter assay. TAZ and Yes-associated protein (YAP) are two closely related transcriptional modulators downstream of the Hippo signaling pathway that is generally activated in numerous types of cancer (31-33). The activation of TAZ and YAP promotes the occurrence and progression of a number of solid tumors via accelerating the proliferation, motility and drug resistance, and inducing the attributes of cancer stem cells $(31,33)$. The essential role of YAP/TAZ in human malignancies suggest the use of YAP/TAZ as potential targets for anticancer drugs $(34,35)$. Furthermore, the roles of Hippo signaling and TAZ in BC have been identified. Hippo signaling exhibits a pivotal role in the development of mammary cancer and the progression of breast cancer (36). TAZ has been reported to accelerate the migration and invasion of BC cells (22). The present study aimed to explore whether TAZ functioned in a circ_0000511/miR-326 axis-mediated regulation of $\mathrm{BC}$ cells. Rescue experiments were performed by transfecting miR-326 alone or together with TAZ overexpression plasmid into $\mathrm{BC}$ cells to test if miR-326 functioned via targeting TAZ. The current results revealed that miR-326 suppressed the proliferation, migration and invasion, and induced apoptosis of BC cells via targeting TAZ. Additionally, it was revealed that circ_0000511 enhanced the abundance of TAZ mRNA and protein via serving as a miR-326 sponge in $\mathrm{BC}$ cells.

According to the current results of in vitro experiments, it was demonstrated that circ_0000511 accelerated the proliferation, migration and invasion, and restrained the apoptosis of BC cells via regulating the miR-326/TAZ axis. The in vivo role of circ_0000511 was explored by building xenograft mice models using MCF-7 cells with the stable silencing of circ_0000511 or control MCF-7 cells. Circ_0000511 interference resulted in the suppression of BC growth in vivo.

The in vivo influence of circ_0000511 on the metastasis of $\mathrm{BC}$ requires to be further explored in future studies. Overall, the present findings revealed that circ_0000511 and TAZ expression was upregulated, while miR-326 expression was downregulated in BC, and circ_0000511 accelerated the progression of $\mathrm{BC}$ by promoting the proliferation, migration and invasion, and impeding the apoptosis of $\mathrm{BC}$ cells via the miR-326/TAZ axis.

\section{Acknowledgements}

Not applicable.

\section{Funding}

No funding was received.

\section{Availability of data and materials}

The datasets used and/or analyzed during the current study are available from the corresponding author on reasonable request.

\section{Authors' contributions}

DW and HJ conceived and designed the study. DW, HJ and $\mathrm{ZZ}$ were responsible for data acquisition and interpretation. HJ and SL performed the statistical analysis. DW and HJ drafted the manuscript. ZZ and SL critically revised the manuscript for important intellectual content. All authors read and approved the final manuscript.

\section{Ethics approval and consent to participate}

The present study involving human samples and animal experiments was approved by the Ethics Committee of The First Hospital of Jilin University (Changchun, China) and was conducted in accordance with the Declaration of Helsinki. All participants provided written informed consent before surgery.

\section{Patient consent for publication}

Not applicable.

\section{Competing interests}

The authors declare that they have no competing interests. 


\section{References}

1. Tao Z, Shi A, Lu C, Song T, Zhang Z and Zhao J: Breast cancer: Epidemiology and Etiology. Cell Biochem Biophys 72: 333-338, 2015.

2. Baselga $\mathrm{J}$ and Swain SM: Novel anticancer targets: Revisiting ERBB2 and discovering ERBB3. Nat Rev Cancer 9: 463-475, 2009

3. Hynes NE and MacDonald G: ErbB receptors and signaling pathways in cancer. Curr Opin Cell Biol 21: 177-184, 2009.

4. Li Y, Zheng Q, Bao C, Li S, Guo W, Zhao J, Chen D, Gu J, He X and Huang S: Circular RNA is enriched and stable in exosomes: A promising biomarker for cancer diagnosis. Cell Res 25: 981-984, 2015.

5. Salzman J: Circular RNA expression: Its potential regulation and function. Trends Genet 32: 309-316, 2016.

6. Sekar S and Liang WS: Circular RNA expression and function in the brain. Noncoding RNA Res 4: 23-29, 2019.

7. Zhou Z, Sun B, Huang S and Zhao L: Roles of circular RNAs in immune regulation and autoimmune diseases. Cell Death Dis 10 : $503,2019$.

8. Greene J, Baird AM, Brady L, Lim M, Gray SG, McDermott R and Finn SP: Circular RNAs: Biogenesis, function and role in human diseases. Front Mol Biosci 4: 38, 2017.

9. Han B, Chao J and Yao H: Circular RNA and its mechanisms in disease: From the bench to the clinic. Pharmacol Ther 187: 31-44, 2018.

10. Lasda E and Parker R: Circular RNAs: Diversity of form and function. RNA 20: 1829-1842, 2014

11. Hansen TB, Jensen TI, Clausen BH, Bramsen JB, Finsen B, Damgaard CK and Kjems J: Natural RNA circles function as efficient microRNA sponges. Nature 495: 384-388, 2013.

12. Panda AC: Circular RNAs act as miRNA sponges. Adv Exp Med Biol 1087: 67-79, 2018.

13. Zhong Y, Du Y, Yang X, Mo Y, Fan C, Xiong F, Ren D, Ye X, Li C, Wang Y, et al: Circular RNAs function as ceRNAs to regulate and control human cancer progression. Mol Cancer 17: 79, 2018.

14. Ding L and Zhang H: Circ-ATP8A2 promotes cell proliferation and invasion as a ceRNA to target EGFR by sponging miR-433 in cervical cancer. Gene 705: 103-108, 2019.

15. Sun H, Xi P, Sun Z, Wang Q, Zhu B, Zhou J, Jin H, Zheng W, Tang W, Cao H and Cao X: Circ-SFMBT2 promotes the proliferation of gastric cancer cells through sponging miR-182-5p to enhance CREB1 expression. Cancer Manag Res 10: 5725-5734, 2018.

16. Bartel DP: MicroRNAs: Genomics, biogenesis, mechanism, and function. Cell 116: 281-297, 2004.

17. Lin S and Gregory RI: MicroRNA biogenesis pathways in cancer. Nat Rev Cancer 15: 321-333, 2015.

18. Ghaemi Z, Soltani BM and Mowla SJ: MicroRNA-326 functions as a tumor suppressor in breast cancer by targeting ErbB/PI3K signaling pathway. Front Oncol 9: 653, 2019.

19. Kanai F, Marignani PA, Sarbassova D, Yagi R, Hall RA Donowitz M, Hisaminato A, Fujiwara T, Ito Y, Cantley LC and Yaffe MB: TAZ: A novel transcriptional co-activator regulated by interactions with 14-3-3 and PDZ domain proteins. EMBO J 19: 6778-6791, 2000.
20. Mahoney WM Jr, Hong JH, Yaffe MB and Farrance IK: The transcriptional co-activator TAZ interacts differentially with transcriptional enhancer factor-1 (TEF-1) family members. Biochem J 388: 217-225, 2005.

21. Murakami M, Tominaga J, Makita R, Uchijima Y, Kurihara Y, Nakagawa $\mathrm{O}$, Asano $\mathrm{T}$ and Kurihara $\mathrm{H}$ : Transcriptional activity of Pax3 is co-activated by TAZ. Biochem Biophys Res Commun 339: 533-539, 2006.

22. Chan SW, Lim CJ, Guo K, Ng CP, Lee I, Hunziker W, Zeng Q and Hong W: A role for TAZ in migration, invasion, and tumorigenesis of breast cancer cells. Cancer Res 68: 2592-2598, 2008.

23. Livak KJ and Schmittgen TD: Analysis of relative gene expression data using real-time quantitative PCR and the 2(-Delta Delta C(T)) method. Methods 25: 402-408, 2001.

24. Shi F, Shi Z, Zhao Y and Tian J: CircRNA hsa-circ-0014359 promotes glioma progression by regulating miR-153/PI3K signaling. Biochem Biophys Res Commun 510: 614-620, 2019.

25. Wan J, Hao L, Zheng X and Li Z: Circular RNA circ 0020123 promotes non-small cell lung cancer progression by acting as a ceRNA for miR-488-3p to regulate ADAM9 expression. Biochem Biophys Res Commun 515: 303-309, 2019.

26. Xu JZ, Shao CC, Wang XJ, Zhao X, Chen JQ, Ouyang YX, Feng J, Zhang F, Huang WH, Ying Q, et al: circTADA2As suppress breast cancer progression and metastasis via targeting miR-203a-3p/SOCS3 axis. Cell Death Dis 10: 175, 2019.

27. Li C, Tian Y, Liang Y and Li Q: Circ_0008035 contributes to cell proliferation and inhibits apoptosis and ferroptosis in gastric cancer via miR-599/EIF4A1 axis. Cancer Cell Int 20: 84, 2020.

28. Qu Y, Zhu J, Liu J and Qi L: Circular RNA circ_0079593 indicates a poor prognosis and facilitates cell growth and invasion by sponging miR-182 and miR-433 in glioma. J Cell Biochem 120: 18005-18013, 2019.

29. Ji S, Zhang B, Kong Y, Ma F and Hua Y: miR-326 Inhibits Gastric Cancer Cell Growth Through Downregulating NOB1. Oncology Pesearch 25: 853-861, 2017.

30. Liang X, Li Z, Men Q, Li Y, Li H and Chong T: miR-326 functions as a tumor suppressor in human prostatic carcinoma by targeting Mucin1. Biomed Pharmacother 108: 574-583, 2018.

31. Moroishi T, Hansen CG and Guan KL: The emerging roles of YAP and TAZ in cancer. Nat Rev Cancer 15: 73-79, 2015.

32. Nguyen CDK and Yi C: YAP/TAZ signaling and resistance to cancer therapy. Trends Cancer 5: 283-296, 2019.

33. Zanconato F, Cordenonsi M and Piccolo S: YAP/TAZ at the roots of cancer. Cancer Cell 29: 783-803, 2016.

34. Warren JSA, Xiao Y and Lamar JM: YAP/TAZ activation as a target for treating metastatic cancer. Cancers (Basel) 10: 115, 2018.

35. Zanconato F, Battilana G, Cordenonsi M and Piccolo S: YAP/TAZ as therapeutic targets in cancer. Curr Opin Pharmacol 29: 26-33, 2016.

36. Shi P, Feng J and Chen C: Hippo pathway in mammary gland development and breast cancer. Acta Biochim Biophys Sin (Shanghai) 47: 53-59, 2015.

c) (i) $\ominus$ This work is licensed under a Creative Commons Attribution-NonCommercial-NoDerivatives 4.0 International (CC BY-NC-ND 4.0) License. 